\title{
FUNCIONAMIENTO SOCIAL DEL ADULTO MAYOR
}

\author{
SOCIAL FUNCTIONING OF ELDERLY \\ MERCEDESZAVALA G.*, DAISY VIDAL G.**, MANUEL CAST RO S.***, \\ PILAR QUIROGA**** y GONZALO KLASSEN P. *****
}

\begin{abstract}
RESUMEN
Estudio descriptivo relacional, destinado a conocer la autoestima y el funcionamiento social de los ancianos según roles sociales, desde su visión y la de sus cuidadores y la percepción del funcionamiento familiar. Se incluyó a 300 ancianos y sus cuidadores familiares, de los consultorios de atención primaria deConcepción. Se aplicó una encuesta en domicilio, con tres escalas: Groningen Social Disabilities Schedule; Escala de Funcionamiento Familiar de Smilkeinstein y el Test deAutoestima de Rosenberg. Resultados: el $51 \%$ de los ancianos reportan una alta autoestima. La percepción de funcionamiento familiar de éstos se ubica en un $84 \%$, lo que correspondea la más alta categoría deesta variable. No se encontró diferencia estadísticamentesignificativa en la evaluación de la percepción de funcionamiento social, entre la autoevaluación del anciano y la evaluación que hace el cuidador respecto del anciano. Es importante destacar una alta consistencia entre ambas mediciones, correlación de un $74 \%$. Respecto a la relación entre las mediciones del autorreporte de funcionamiento social de los ancianos y la escala de autoestima, se encontró una correlación positiva, a mejor funcionamiento social obtenido del autorreporte, mayor autoestima. Lo mismo ocurre entre las variables autorreporte de Funcionamiento social de los ancianos y la Escala de Percepción de funcionamiento familiar-Apgar. Los resultados de esta investigación podrán ser considerados por los profesionales de salud para que en el trabajo con ancianos y sus familias se fomente la independencia, la funcionalidad y la calidad de vida.
\end{abstract}

Palabras claves: Anciano, familia, autoestima, funcionamiento social, cuidadores.

\begin{abstract}
ABST RACT
Relational descriptive study aimed to asses elderly self esteem and social functioning according to the social roles, through their personal and caregiver perceptions and the family functional perception. The sampleincluded 300 elders and the caregivers from Primary Care Centers in the city of Concepción, Chile. It was applied an inquest at home with 3 scales: the Groningen Social Disabilities Schedule; the Smilkeinstein Family Functioning scaleand the Rosen berg self esteem test. Results: $51 \%$ of elders and $84 \%$ report a high self esteem. The functioning familiar perception of them is at $84 \%$, that corresponds to a high level of perception. It was not found estatistically significant difference in social functioning perception between self esteem of elder and the evaluation made by the caregiver. It is important to say that is a high consistence between both measures. Correlation of $74 \%$. About the relation between the self social function report of elders and self esteem scale, it was founded a positive correlation. The better the social functioning in the self report, the better the self esteem. The same phenomenon was observed between the elders self reporting social functioning and theAPGAR family functioning perception
\end{abstract}

\footnotetext{
* Enfermera, Mg. en Enfermería, docente Depto. de Enfermería, Facultad de Medicina, Universidad de Concepción, Concepción, Chile. E-mail: mzavala@udec.cl

** Asistente Social, M g. en Salud Pública. DocenteD epto. de Servicio Social, Facultad de Ciencias Sociales, Universidad deConcepción, Concepción, Chile. E-mail: dvidal@udec.cl

*** Enfermero, M g. en Enfermería, docente D epto. de Enfermería, Facultad de M edicina, Universidad de Concepción, Concepción, Chile. E-mail: mcastro@udec.cl

**** M édico, especialista en Psiquiatría, docente Depto. Educación M édica, Facultad de M edicina, Universidad de Concepción, Concepción, Chile. E-mail: quirogal@vtr.net

***** M édico, especialista en Neurología, docenteDepto. de Especialidades, Facultad de M edicina, Universidad deConcepción, Concepción, Chile. E-mail: gklaasse@udec.cl
} 
scale. The results of this study could be considered by health professionals so that in working with elders and theirs families, the independence, the functioning and quality of life, could be increased

Keywords: Elders, family, self esteem, caregiver, social functioning.

Fecha de recepción: 16/05/06. Fecha aceptación: 09/11/06

\section{INTRODUCCION}

El envejecimiento concebido como un proceso que experimentan los individuos puede ser visto desde dos enfoques, uno biológico y otro social. Para el profesor Thomae (1982), la vejez es un destino social, puesto que son las características de la sociedad las que condicionan tanto la cantidad, como la calidad de vida. Peter Thownsend, citado por Barros (1993) plantea algo similar cuando sostiene que la situación actual de dependencia de los ancianos ha sido creada por la política social. Una política social en muchas ocasiones no publicada.

Chawla (1988) afirma quelosancianos"no si empre son dependientes a causa de su decadencia física, sino que lo son frecuentemente debido a la forma cómo son categorizadossocialmente y a las presiones que se ejercen sobre ellos. Si bien es cierto que el envejecer implica un deterioro biológico, son las condiciones sociales decarencia de servicios y lasideas contenidas en el modelo médico tradicional las que restan oportunidades para queel "viejo" se mantenga activo y autovalente. La carencia de una definición sociocultural del conjunto de actividades que serían específicas de losancianos les hace sentirse inútiles y sin reconocimiento social. La falta de tareas específicas conlleva la dificultad para saber en qué concentrar los esfuerzos y en qué volcarse de modo de actualizar las propias potencialidades. Cada individuo que envejece debe crearse un personaje eimprovisar su actuación: la dificultad de esto implica que sólo algunos lo logran y otros deben resignarse al estrecho mundo de las cuatro paredes del hogar (Barros, 1994; Anzola et al., 1994).
Chilese encuentra en un proceso deenve jecimiento poblacional paulatino, lo que se traduce en que en el año 2000, el 10,2\% del total de la población del país estaba compuesta por personas de 60 años y más de edad, esto es 1.521.557 personas, proyectándose a un 18,2\% para el año 2025, con una población superior a los 3 millones y medio (CELADE, 2003).

El aumento progresivo de las expectativas de vida en todas las sociedades presenta para los próximos decenios el importante desafío de definir el significado y los roles sociales de los adultos mayores. Este desafío implica la necesidad de realizar arreglos sociales que permitan a las personas mayores el desarrollo de todas sus potencialidades en busca de un envejecimiento exitoso. Un envejecimiento exitoso y saludabletienequever con la funcionalidad biológica y social que permite la plena integración social y familiar de los que envejecen, lo que implicará superar las barreras sociales y culturales que aún persisten e impiden a losadultos mayores desarrollar sus potencialidades. En las modificaciones que deban realizarse están involucrados el estado, las organizaciones sociales, la comunidad, la familia y cada uno de los individuos (Peláez, 2000; Domínguez, 1982).

El principal sistema social del anciano y el apoyo que recibe viene de su familia. Se trata de una unidad social sujeta a las presiones y condiciones desu entorno cultural, económico y político deun momento dado. La definición más aceptada del concepto de familia es la de varias personas relacionadas por los lazos de parentesco, sanguíneos, de matrimonio o por adopción. Tales personas pueden compartir un mismo techo o habitar en viviendas separadas. 
La familia del anciano brinda apoyo social, funcional, económico o material, afectivo y asistencia en diversas formas.

Aun cuando la gran mayoría de las personas adultas mayores son relativamente independientes y físicamente aptas y funcionales, el grupo de los mayores de 80 son más dependientes, más aún si su nivel socioeconómico y estado de salud son limitados (Sánchez, 1994). La O.M.S. ha definido la salud del anciano por su capacidad para funcionar, y no por las limitaciones, discapacidad o enfermedad que presente.

Así, la calidad de vida de las personas mayores está estrechamente relacionada con la capacidad funcional y el conjunto de condiciones que le permiten mantener su participación en su propio autocuidado y en la vida familiar y social (CELADE, 2003; FernándezBallesteros et al., 1996).

Lainteracción delosfactores biológicoscon la psicología del comportamiento y con los procesos sociológicos del envejecimiento aparececon frecuencia en los estudios realizados a la fecha. La posibilidad de controlar una enfermedad está condicionada por la aptitud social para integrar a la persona enferma a un sistema de vida que le ayude a resolver sus problemas ambientales y particularmente la aptitud para desenvolverse en la actualidad. Deigual forma, la capacidad social para integrarse varía según el individuo que envejece. (O.P.S./OM S, 1985)

La tendencia a favor de una vejez activa procura que el anciano conservesu independencia, su libertad de acción y su iniciativa, de manera que sea capaz de elegir el mayor número de opciones en su vida, sin desconocer la realidad de que la gente de mayor edad es menos capaz de ejercer su independencia, tiene menos eficiencia en su trabajo y es más vulnerablea las enfermedades (Barros, 1994).

La persona mayor ha de tomar una actitud positiva en la vida y la primera de estas actitudes básicas es aprender a ser uno mismo, aprender a saber vivir consigo mismo, a co- nocerse tal y como uno es, con sus dimensiones reales, espaciales, temporales, corporales, espirituales.

Esta expresión de JoséM . Cagigal sintetiza todos aquellos puntos necesarios para una reconceptualización y revalorización de la edad adulta tardía como una etapa normal de la vida del ser humano, con sus características propias y definidas y que llevan inmersa la posibilidad deprepararse para la vejez. Esto implica esencialmente, y a modo de síntesis, asumir y resolver la aceptación dela edad, una redefinición de roles, la búsqueda e identificación de formas alternativas para el cese de ingresos y, la revisión de vida y establecimiento denuevas metas. Entrega un sello especial, propio del ambiente sociocultural en el cual se inserta. Así, el "funcionamiento" de una persona en sociedad es factible de medir a través de su desempeño, en los distintos roles sociales que le corresponde ejecutar (Parsons, 1999). El concepto de rol social ha sido definido de diferentes maneras. En este estudio se considera la opción de la integración de los conceptos de rol social y rol conductual. "Rol social es un conjunto de expectativas que las personas tienen acerca de la conducta de una persona, quien toma o adopta una posición determinada" (O.P.S./O.M.S., 1985). Se puede precisar queel papel que una persona desempeña en sociedad está influido por las nociones propias y expectativas acerca de cómo debe comportarse; las nociones y expectativas de otros relevantes, la norma del grupo 0 de la población; las sanciones positivas o negativas que imponen otras personas (y posiblementeel mismo individuo), cuando el rol de conducta relevante se encuentra en discusión. El desempeño de un rol social determinado adquiere gran relevancia, por cuanto es el mecanismo que permite la integración de una persona al sistema social en el cual se inserta. Para medir estas dimensiones seha considerado la mirada que otorga el Groningen Social Disabilities (Wiersma et al., 1990). 


\section{OBJETIVOS DEL ESTUDIO}

Objetivo general 1

- Conocer el funcionamiento social delosadultos mayores de la comuna de Concepción según algunas dimensiones psicosocioculturales y familiares que lo caracterizan (roles sociales, autoestima y percepción defuncionamiento familiar).

\section{Objetivo general 2}

- Contrastar el autorreporte del funcionamiento social del adulto mayor y la percepción de funcionamiento que tiene de él, un cuidador, a través de la medición del Groningen.

\section{MATERIAL Y METODO}

El presente trabajo es un estudio descriptivo relacional, cuya técnica usada fue el survey social.

Las unidades de análisis estuvieron constituidas por:

a) Los ancianos inscritos en el Programa del Adulto M ayor de los consultorios de atención primaria dela comuna deConcepción $\mathrm{y}$

b) Los cuidadores de estos ancianos.

El universo estuvo constituido por 4.162 adultosmayoresinscritosen el ProgramaAdulto $\mathrm{M}$ ayor delos Consultorios deAtención Primaria dela Comuna deConcepción. Seutilizó un muestreo por cuotas, seleccionando a los sujetos conforme cumplían lossiguientes criterios: ancianos de ambos sexos mayores de 60 años para mujeres y mayores de 65 para varones; vivir con un cuidador familiar; no presentar alteraciones mentales, esto fue verificado mediantela aplicación del minimental test; ser autovalente, de acuerdo a la escala de funcionalidad de la Cruz Roja española.
La muestra quedó finalmente conformada por 300 ancianos y 300 cuidadores que cumplían los requisitos anteriormente señalados.

\section{Del instrumento}

Para la obtención de los datos se elaboró una encuesta para el anciano y otra para el cuidador.

La encuesta del anciano incluyó

I. Antecedentes sociodemográficos.

II. Evaluación del funcionamiento familiar a través del test deApgar familiar de Smilkstein, adaptado por Horwitz y Florenzano (1985). Este instrumento evalúa la percepción de funcionamiento familiar según la persona que responde.

III. Test de Autoestima de Rosemberg; Escala utilizada para la medición global de la autoestima, incluyediezítemes cuyos contenidos se centran en los sentimientos de respeto y aceptación de sí mismo/a. La mitad de los itemes están enunciados positivamente y la otra mitad negativamente (Vázquez et al., 2004).

IV. Evaluación de funcionamiento social de las discapacidades sociales, Groningen II versión (Wiersma et al., 1990). "Groningen Social Disabilities Schedule", de ahora en adelante: G.S.D.S. II (Segunda versión) traducido al castellano como "Registro Groningen de las Discapacidades Sociales". Sebasa en la descripción deocho roles sociales, los que de acuerdo con la revisión teórica y conceptual realizada por el equipo creador del instrumento eran los que permitían medir, adecuadamente, el funcionamiento social de una persona. Los roles contemplados en esa clasificación son: Rol de cuidado personal; Rol de la familia; Rol del parentesco; Rol de pareja; Rol de padre; Rol de ciudadano; Rol social y Rol ocupacional. 
La encuesta del cuidador incluyó:

I. Antecedentes básicos de identificación; II. Evaluación de funcionamiento social de las discapacidades sociales, Groningen II versión (Wiersma et al., 1990), que consideró la percepción del cuidador respecto del anciano.

\section{RESULTADOSY DISCUSION}

\section{Características sociodemográficas de los Ancianos ( Tabla I)}

La información quese presenta en Tabla I indica que el $68 \%$ de la muestra es de sexo femenino, en la categoría de 70 a 79 años se concentra el mayor porcentaje (48,3\%) delos ancianos encuestados, lo que coincide con el grupo de edad, de este segmento de la población, que está creciendo con mayor rapidez. En cuanto al estado civil, un $49,7 \%$ son casadosy un 37,3\% informan ser viudos. La escolaridad de los ancianos se concentra en las categorías sin escolaridad y escolaridad básica incompleta, este hecho da cuenta de la realidad sociocultural que esta población vivió durante su infancia, y responde también al perfil de la población que se atiende en el sistema público de salud.

Variables Psicosociales Ancianos: Autoestima; Percepción de Funcionamiento Familiar; Funcionamiento Social; Percepción de Funcionamiento social delos cuidadores respecto de los ancianos (Tabla II).

De acuerdo a lo que se indica en Tabla $\mathrm{N}$ o 2 , un $53 \%$ de los ancianos se ubica en el nivel de alta autoestima, sólo un 2,6\% resulta calificado con una baja autoestima. Respecto a la percepción de funcionamiento familiar, el $84 \%$ de los ancianos reconoce una alta percepción de funcionamiento familiar, sólo el $4,6 \%$ percibeel funcionamiento de su familia como deficiente-bajo. En relación al funcio- namiento social, el 90,7\% delos ancianosencuestados reporta un buen funcionamiento social. Final mente, y respecto de la percepción que tiene el cuidador acerca del funcionamiento social del anciano, un $87,6 \%$ de ellos ubica a los ancianos en la categoría de adecuado funcionamiento social.

\section{Puntaje Groningen del anciano según áreas de desempeño (Tabla IV)}

De acuerdo con lo que indica la Tabla III, las mayores frecuencias se agrupan siempre en las categorías "Funcional" (más del $50 \%$ delas frecuencias, a excepción del área Rol de Pareja con sólo un $31 \%$ en esta categoría) y en segunda prioridad en la categoría D isfuncionalidad Leve (sobre el $15 \%$ de las frecuencias, exceptuando nuevamenteel Area Rol de Pareja que aglutina sólo un $8 \%$ de respuestas en esta categoría).

Los roles que destacan con un mejor funcionamiento social son: Rol deAutocuidado, Rol de Familia y Rol O cupacional.

Sin perjuicio delo anterior, lasáreas queel anciano identifica con mayor dificultad de desempeño 0 aquellas en las que él identifica con un menor funcionamiento social son: Area Rol de Parentesco, Area Rol de Pareja, Area Rol deCiudadano y Area Rol Social. En todas ellas, menos deun $50 \%$ secalifica como "Funcional" (en las otras áreas sobre el $64 \%$ se califica"Funcional", un porcentajecercano al $9 \%$ secalifica en Disfuncionalidad M oderada (en las otras áreas un porcentaje cercano sólo al $5 \%$ secalifica con Disfuncionalidad M oderada) y más del $2 \%$ en Disfuncionalidad Severa. Es importante destacar que estas cuatro áreas hacen alusión a las relaciones interpersonales que mantiene el anciano con sus significativos cercanosy con el grado deconexión con su entorno. 
Tabla I. Características socio-demográficas del anciano.

\begin{tabular}{|l|r|c|}
\hline Variables sociodemográficas & Fi (n=300) & $\%$ \\
\hline * Sexo & 204 & 68 \\
Femenino & 96 & 32 \\
Masculino & & \\
* Edad (años) & 78 & 26 \\
$60-69$ & 145 & 48,3 \\
$70-79$ & 68 & 22,7 \\
$80-89$ & 9 & 3 \\
90 y más & & \\
* Estado civil & 30 & 10 \\
Soltero & 149 & 49,7 \\
Casado & 2 & 0,7 \\
Conviviente & 112 & 37,3 \\
Viudo & 7 & 2,3 \\
Separado & & \\
* Escolaridad & 45 & 15 \\
Sin escolaridad & 150 & 50 \\
Ens. Bás. incomp. & 27 & 9 \\
Ens. Bás. completa & 37 & 12,3 \\
Ens. M edia incomp. & 20 & 6,6 \\
Ens. M edia completa & 5 & 1,7 \\
Ens. Tec. Prof. incomp. & 11 & 3,7 \\
Ens. Tec. Prof. comp. & 5 & 1,7 \\
No responde & & \\
\hline
\end{tabular}

Fuente: Funcionamiento social del adulto mayor. Proyecto DIUC: № 98.082.019-1.0.

Tabla II. Variables psicosociales respecto del anciano.

\begin{tabular}{|l|r|c|}
\hline Puntaje Escala Autoestima & Fi & $\%$ \\
\hline Bajo (2-13) & 8 & 2,7 \\
Medio (14-26) & 133 & 44,6 \\
Alto (27-40) & 157 & 52,7 \\
\hline Total & $(\mathrm{n}=298)$ & 100 \\
\hline Puntaje Apgar & $\mathrm{Fi}$ & $\%$ \\
Bajo (-3) & 14 & 4,7 \\
Medio (4-6) & 33 & 11 \\
Alto (7-10) & 253 & 84,3 \\
\hline Total & 300 & 100 \\
\hline Puntaje Groningen (desde Anciano) & $\mathrm{Fi}$ & $\%$ \\
Funcional (0) & 272 & 90,7 \\
Disfuncionalidad leve (1) & 25 & 8,6 \\
Disfuncionalidad moderada (2) & 0 & 0 \\
Disfuncionalidad severa(3) & 2 & 0,7 \\
\hline Total & 300 & 100 \\
\hline
\end{tabular}

Fuente: Idem Tabla I. 
Funcionamiento social del adulto mayor / M . ZAVALA G. ET AL.

Tabla III. Puntaje Groningen del anciano desdela percepción del cuidador.

\begin{tabular}{|l|r|c|}
\hline Puntaje Groningen & Fi & $\%$ \\
\hline Funcional (0) & 263 & 87,7 \\
Disfuncionalidad leve (1) & 36 & 12 \\
Disfuncionalidad moderada (2) & 1 & 0,3 \\
Disfuncionalidad severa (3) & 0 & 0 \\
\hline Total & 300 & 100 \\
\hline
\end{tabular}

Fuente: Idem Tabla 1.

Tabla IV. Puntaje Groningen entregado por el anciano por áreas de desempeño.

\begin{tabular}{|c|c|c|c|c|c|c|c|c|c|c|c|c|c|c|c|c|}
\hline $\begin{array}{l}\text { Puntaje Groningen } \\
\text { Rol Social Desempeñado }\end{array}$ & \multicolumn{2}{|c|}{$\begin{array}{c}\text { Rol } \\
\text { Autocuid. }\end{array}$} & \multicolumn{2}{|c|}{$\begin{array}{c}\text { Rol } \\
\text { Familia }\end{array}$} & \multicolumn{2}{|c|}{$\begin{array}{c}\text { Rol } \\
\text { Parentesco }\end{array}$} & \multicolumn{2}{|c|}{$\begin{array}{c}\text { Rol } \\
\text { de Pareja }\end{array}$} & \multicolumn{2}{|c|}{$\begin{array}{c}\text { Rol } \\
\text { de Padres }\end{array}$} & \multicolumn{2}{|c|}{$\begin{array}{c}\text { Rol } \\
\text { Ciudadano }\end{array}$} & \multicolumn{2}{|c|}{$\begin{array}{l}\text { Rol } \\
\text { Social }\end{array}$} & \multicolumn{2}{|c|}{$\begin{array}{l}\text { Rol } \\
\text { Ocupac. }\end{array}$} \\
\hline Funcional (0) & 237 & 79,0 & 235 & 78,3 & 171 & 57 & 93 & 31 & 192 & 64 & 167 & 55,7 & 148 & 49,3 & 211 & 70,3 \\
\hline $\begin{array}{l}\text { Disfuncionalidad } \\
\text { leve (1) }\end{array}$ & 44 & 14,7 & 46 & 15,3 & 66 & 22,0 & 25 & 8,3 & 68 & 22,7 & 81 & 27 & 84 & 28 & 64 & 21,3 \\
\hline $\begin{array}{l}\text { Disfuncionalidad } \\
\text { moderada (2) }\end{array}$ & 16 & 5,3 & 14 & 4,7 & 26 & 8,7 & 30 & 10,0 & 19 & 6,3 & 41 & 13,7 & 54 & 18 & 15 & 5,1 \\
\hline $\begin{array}{l}\text { Disfuncionalidad } \\
\text { severa (3) }\end{array}$ & 3 & 1,0 & 5 & 1,7 & 8 & 2,7 & 6 & 2 & 6 & 2,0 & 10 & 3,3 & 10 & 3,3 & 9 & 3,0 \\
\hline No contesta (8) & 0 & 0 & 0 & 0 & 4 & 1,3 & 0 & 0 & 0 & 0 & 0 & 0 & 4 & 1,3 & 1 & 0,3 \\
\hline No corresponde (9) & 0 & 0 & 0 & 0 & 25 & 8,3 & 146 & 48,7 & 15 & 5,0 & 1 & 0,3 & 0 & 0 & 0 & 0 \\
\hline
\end{tabular}

Fuente: Idem Tabla I.

\section{Puntaje Groningen entregado por e cuidador respecto del anciano según áreas de desempeño (Tabla V)}

De acuerdo con lo que indica la Tabla IV, las mayores frecuencias se agrupan siempre en las categorías: Funcional (más del $50 \%$ de las frecuencias, a excepción del Area Rol de Pareja con sólo un $31 \%$ en esta categoría)y en segunda prioridad en la categoría Disfuncionalidad Leve (sobreel $12 \%$ delasfrecuencias, exceptuando nuevamente el Area Rol de Pareja que aglutina sólo un $11 \%$ de respuestas en esta categoría). Las áreas que destacan con un mejor funcionamiento son: Autocuidado, Familia y Ocupacional. Sin perjuicio de lo anterior, las áreas que el cuidador identifica con mayor dificultad de desempeño respecto del anciano, 0 aquellas en las que lo identifica con un menor funcionamiento social son: Area rol de Parentesco, Area Rol de Pareja, Area rol de Ciudadano y Area Rol Social. En todas ellas, menos de un $50 \%$ califica al anciano como "Funcional" (en las otras áreas sobre el $70 \%$ lo califica "Funcional" (exceptuando el Rol de Pareja).

Esimportante destacar que aun cuando la distribución de frecuencias por áreas de desempeño más deterioradas son coincidentes en las mediciones que entrega el anciano con las que entrega su cuidador, los puntajes entre gados por el cuidador se concentran más en 
TablaV. Puntaje Groningen entregado por el cuidador respecto del anciano por áreas de desempeño.

\begin{tabular}{|c|c|c|c|c|c|c|c|c|c|c|c|c|c|c|c|c|}
\hline \multirow{2}{*}{$\begin{array}{l}\text { Puntaje Groningen } \\
\text { Rol Social Desempeñado } \\
\text { Funcional (0) }\end{array}$} & \multicolumn{2}{|c|}{$\begin{array}{c}\text { Rol } \\
\text { Autocuid. }\end{array}$} & \multicolumn{2}{|c|}{$\begin{array}{c}\text { Rol } \\
\text { Familia }\end{array}$} & \multicolumn{2}{|c|}{$\begin{array}{c}\text { Rol } \\
\text { Parentesco }\end{array}$} & \multicolumn{2}{|c|}{$\begin{array}{c}\text { Rol } \\
\text { de Pareja }\end{array}$} & \multicolumn{2}{|c|}{$\begin{array}{c}\text { Rol } \\
\text { de Padres }\end{array}$} & \multicolumn{2}{|c|}{$\begin{array}{l}\text { Rol de } \\
\text { Ciudadano }\end{array}$} & \multicolumn{2}{|c|}{$\begin{array}{l}\text { Rol } \\
\text { Social }\end{array}$} & \multicolumn{2}{|c|}{$\begin{array}{l}\text { Rol } \\
\text { Ocupac. }\end{array}$} \\
\hline & 242 & 80,7 & 227 & 75,7 & 168 & 56 & 93 & 31 & 210 & 70 & 156 & 52,0 & 150 & 50,0 & 220 & 73,3 \\
\hline $\begin{array}{l}\text { Disfuncionalidad } \\
\text { leve(1) }\end{array}$ & 38 & 12,7 & 39 & 13,0 & 79 & 26,3 & 32 & 10,7 & 47 & 15,7 & 94 & 31,3 & 89 & 29,7 & 58 & 19,3 \\
\hline $\begin{array}{l}\text { Disfuncionalidad } \\
\text { moderada (2) }\end{array}$ & 13 & 4,3 & 25 & 8,3 & 19 & 6,3 & 23 & 7,7 & 24 & 8,0 & 41 & 13,7 & 45 & 15,0 & 16 & 5,4 \\
\hline $\begin{array}{l}\text { Disfuncionalidad } \\
\text { severa (3) }\end{array}$ & 7 & 2,3 & 4 & 1,3 & 4 & 1,3 & 8 & 2,7 & 3 & 1,0 & 9 & 3,0 & 9 & 3,0 & 6 & 2,0 \\
\hline No contesta ( 8 ) & 0 & 0 & 0 & 0 & 3 & 1,0 & 2 & 0,7 & 0 & 0 & 0 & 0 & 6 & 2,0 & 0 & 0 \\
\hline No corresponde ( 9 ) & 0 & 0 & 5 & 1,7 & 27 & 9,0 & 142 & 47,3 & 16 & 5,3 & 0 & 0 & 1 & 0,3 & 0 & 0 \\
\hline
\end{tabular}

Fuente: Idem Tabla V.

las categorías 1, "Funcional", las mediciones que entrega el anciano se agrupan de igual forma en categoría 1, pero dispersan también más puntajes en las categorías D isfuncionalidad M oderada y Disfuncionalidad Severa.

\section{CONCLUSIONES}

I. Los ancianos inscritos en el Programa del Adulto Mayor de los Consultorios de Atención Primaria de la Comuna de Concepción responden al siguiente perfil: $75 \%$ de ellos son del sexo femenino, el $48,3 \%$ se distribuyen entre las edades de 70 a 79 años, destacándose un $22,6 \%$ de 80 y más años, y un $3 \%$ de más de 90 años; el $50 \%$ de ellos son casados, manteniéndose un $10 \%$ del grupo en la categoría solteros; su escolaridad es baja, un $65 \%$ no tieneescolaridad o tiene enseñanza básica incompleta; el 70,6\% de ellos se declaran católicos; respecto de las variables socioeconómicas de los ancianos se puede destacar que: el mayor porcentaje de los ancianos encuestados seubica en la categoría dejubilados, con un $38 \%$. El $61 \%$ de los ancianos son cotizantes del Instituto de Normalización Previsional (INP); respecto de su previsión en salud, el
94\% de ellos perteneceal Fondo Nacional de Salud. En cuanto a la actividad desempeñada por el adulto mayor, previo a la jubilación, el $33 \%$ de ellos se desempeñó como empleado u "obrero". En la actualidad el 91,3\% no desempeña actividad laboral remunerada.

II. Respecto de las variables psicosociales investigadas, es importante destacar:

Autoestima: la mayor frecuencia delosancianos se ubica en la categoría alta autoestima con un $52,6 \%$, sólo un $2,6 \%$ seubica en la categoría baja autoestima. Este resultado tie ne correlación con el puntaje Groningen reportado por el anciano, mientras el anciano se percibe con una alta autoestima, mejor es también su autorreporte en funcionamiento social.

Respecto dela percepción defuncionamiento familiar, un $84,3 \%$ califica en la categoría "alto", es decir, percibe como altamente positivo el funcionamiento de su familia. Esteresultado es también coincidente con el autorreporte en funcionamiento social, a mejor percepción de funcionamiento familiar social informado, mejor desempeño en funcionamiento social. 
III. Al contrastar el autorreporte del adulto mayor y la percepción que de él tiene su cuidador, respecto de funcionamiento social, los resultados permiten afirmar que existe una alta correlación entreambas mediciones, $74 \%$. Aun cuando el autorreporte del anciano fue levemente superior a lo informado por su cuidador, no se obtienen diferencias estadísticamente significativas.

Tanto para el puntaje Groningen del anciano como para el puntaje Groningen del cuidador, las mayoresfrecuencias seubicaron en la categoría "Funcional", 90,7\% y 87,6\% respectivamente.

IV. Analizando el puntaje Groningen por áreas de desempeño, tanto para el puntaje Groningen del anciano como para el puntajeGroningen del cuidador, estos resultados fueron altamente coincidentes. Las áreas identificadas con mayor dificultad de desempeño para ambos casos son las de Parentesco, de Pareja, deCiudadano y Social, todas ellas en conexión con relaciones interpersonales que mantiene el anciano con sus cercanos significativos y con el grado de conexión que mantiene con su entorno.

V. El hecho que los ancianos califiquen con alta Autoestima una adecuada percepción de funcionamiento familiar y sobretodo con un buen funcionamiento social en las ocho áreas estudiadas, cambia la perspectiva que la sociedad y principalmente la de los profesionales de la salud que trabajan con ellos, tienen. Esto debiera permitir trabajar programas de autocuidado, recreación y actividadesgrupales, lo que a su vez mantiene a los ancianos con una actividad social, mejora su salud física y mental y su calidad de vida (D arton-Hill, 1997).

Para la familia, principal cuidadora del anciano, se le facilita la tarea de cuidar, aun cuando es importante desarrollar programas que los integren fuertemente al trabajo realizado con el anciano.

Es también importante destacar que en la medida que el anciano mantenga su autova- lencia y un adecuado rol social incidirá en la mantención deuna buena salud física y mental. En la medida queel anciano mantenga un rol social se convierte cada vez más en un ser útil y de gran valor para sus significativos cercanos y por ende se facilita también la tarea del cuidador.

\section{REFERENCIAS BIBLIOGRAFICAS}

Anzola Pérez, E.; Galinsky, D.; M orales, M.F.; Salas, A. y Sánchez, M. (1994). La atención de los ancianos: Un desafío para los años noventa. Publicación científica 546. Organización Panamericana de la Salud.

Barros, C. (1993). Factores que intervienen en el bienestar de los adultos mayores. Estudios Sociales № 77.

------ . (1994). Aspectos sociales del envejecimiento en la atención delosancianos. Un desafío para los años noventa. Publicación Científica O.P.S. Washington.

Chawla, T. (1998). The Participation of the Elderly in Development. United Nations, CSDHA, International Seminar Expert Group.

Centro Latinoamericano y Caribeño de Demografía. CELADE (1990). División de Población de IaCEPAL. Area de Población y Desarrollo. Lasituación de las personas mayores. Santiago de Chile.

Centro Latinoamericano y Caribeño de Demografía. CELADE (2003). División de Población de la CEPAL. La situación de las personas mayores. Santiago de Chile.

Darton-Hill, I. (1997). El envejecimiento con salud y calidad de vida. Salud para todos. Mensuario de salud y acción social. Año 5, № 47. Bs. As, Argentina.

Domínguez, O. (1982). La vejez nueva edad social. Editorial Andrés Bello. Santiago de Chile.

Fernández-Ballesteros, R.; Zamarron, M y y arcia, A. (1996). Calidad de vida en la vejez en los distintos contextos. Documentos Técnicos № 83. Universidad Autónoma deM adrid. Instituto $\mathrm{Na}$ cional de Servicios Sociales (Inserso). M inisterio de Trabajo y Asuntos Sociales, M adrid, España.

Horwitz, N. y Florenzano, R. (1985). Familiay salud familiar: Un enfoque para la atención primaria. Boletín OPS No 98 (2).

OPS/OMS (1985). Hacia el bienestar de los ancianos. Publicación Científica № 492. Washington D.C.U.S.A. 
Parsons, Talcott (1999). El sistema social. Versión de José Jiménez Blanco y José Cazorla Pérez. Madrid: Alianza.

Peláez, M. (2000). Organización Panamericana de la Salud. Programa de Salud Familiar. Washington D.C.

Sánchez A., M elba (1994). El apoyo social informal, en "La atención de los ancianos. un desafío para Ios noventa". O.P.S./ O.M. S. Publicación Científica 546.

Thomae H. (1982). New Perspectives on Old Age. New York: Springer Publishing Co.
Vázquez, A.J.; Jiménez, R. y Vázquez, R. Escala de autoestima de Rosenberg: Fiabilidad y validez en población clínica española. Apuntes de Psicología Colegio Oficial de Psicología. 2004, Vol. 22, № 2, pp. 247-255. de Andalucía Occidental y ISSN 0213-3334 Universidad de Sevilla.

Wiersma, D.; Dejong, A.; Ormel, J. \& Kraaijkamp, H. (1990). G.S.D.S. II Segunda versión. The Groningen Social Disabilities Schedule: M anual For The Use Of The Instrument For Assessing Disabilities In Social Functioning. Departament Of Social Psychiatric, University Of Groningen. Holanda. 